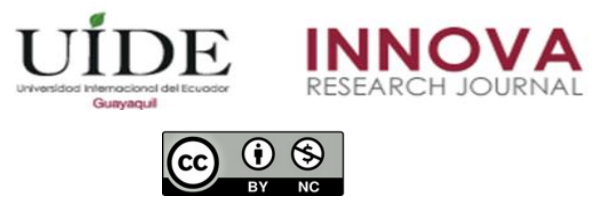

INNOVA Research Journal, ISSN 2477-9024

(Enero-Abril 2020). Vol. 5, No.1 pp. 255-266

DOI: https://doi.org/10.33890/innova.v5.n1.2020.1240

URL: http://revistas.uide.edu.ec/index.php/innova/index

Correo: innova@uide.edu.ec

\title{
Los tres pilares para la retención del talento humano en una empresa de servicios generales
}

\section{The three pillars for the retention of human talent in a general services company}

Fernando Alexis Nolazco Labajos

https://orcid.org/0000-0001-8910-222X

Diego Anthony Rodríguez Huancahuari

https://orcid.org/0000-0002-5803-7768

Universidad Norbert Wiener, Perú

Autor para correspondencia: fernandonolazco@gmail.com; diegoanthony011@gmail.com

Fecha de recepción: 18 de octubre del 2019 - Fecha de aceptación: 07 de enero del 2020

\section{Resumen}

El presente trabajo de investigación tuvo por objetivo proponer los tres pilares para reducir la rotación del talento humano. La metodología utilizada se basó en el sintagma holístico, enfoque mixto, tipo proyectiva, nivel comprensivo. Para obtener la información se realizó un análisis documentario del "Seguro complementario de trabajo de riesgo" del periodo 2015-2018 y se efectuó entrevistas a siete personas, de los cuales tres fueron ex trabajadores, tres trabajadores que laboran actualmente y al gerente general de la empresa. Se determinó que la rotación del personal en el año 2015 fue en promedio el 123\%, mientras que en el año 2016 el 99\%, el 2017 fue $91 \%$ y el 2018 el 50\% en promedio. Por tal razón se esbozó los tres pilares para la retención del talento humano, el primer pilar corresponde a la implantación de un nuevo proceso de contratación del personal con el fin de optimizar el tiempo, el segundo pilar atañe a la creación del perfil de los puestos de trabajo, con el fin de contratar personal idóneo, finalmente, el tercer pilar incumbe al plan de involucramiento del personal, todo lo anterior permitirá cumplir los objetivos de la organización.

Palabras claves: rotación del personal; talento humano; satisfacción laboral; seguridad laboral; puestos de trabajo; compromiso; proceso de contratación

\begin{abstract}
The present research work was to propose the three pillars to reduce the rotation of human talent. The methodology used was based on the holistic syntagma, mixed approach, projective type, comprehensive level. In order to obtain the information, a documentary analysis of the "Supplementary work risk insurance" for the period 2015-2018 and was carried out, in turn, interviews were carried out with seven people, of which three were former workers, three workers who currently work and the general manager of the company. It was determined that the turnover of personnel in 2015 was on average 123\%, while in $201699 \%, 2017$ was $91 \%$ and $201850 \%$ on average. For this reason, the three pillars for the retention of human talent were
\end{abstract}


outlined, the first pillar corresponds to the implementation of a new process of hiring of the personnel in order to optimize time, the second pillar concerns the creation of the profile of the posts of work, in order to hire qualified staff, finally, the third pillar concerns the plan of involvement of the staff, all the above will allow to meet the objectives of the organization.

Key words: staff turnover; human talent; job satisfaction; job security; jobs; commitment; hiring process

\section{Introducción}

En el mundo globalizado, donde es exigente la competitividad institucional y empresarial, se manifiesta el talento humano como el escalón para lograr el éxito, considerado como la suma de las habilidades y capacidades potenciadas del sujeto en beneficio de su contexto.

Lamentablemente con el pasar del tiempo algunas compañías aún persisten en no considerar importante la satisfacción de su personal, estancando la demostración del máximo potencial que pueden ofrecer sus trabajadores. Solo se preocupan que exista un salario aceptable que garantice que los colaboradores no abandonen sus puestos de empleo hasta que la empresa lo determine.

El trato amable y empático, así como brindarles la posibilidad de un desarrollo profesional con una línea de carrera en lo que mejor se desenvuelve, es la exigencia que toda empresa debe considerar para mantener a sus empleados satisfechos, complacidos y motivados. Diversos estudios indicaron que las compañías que presentaron una rotación de su personal inferior al cinco por ciento en los sectores industriales y financieros, consideraron que el factor remuneración fue la principal causa para que los empleados opten por cambiar de trabajo. La segunda posición fue la falta de oportunidades para crecimiento profesional y por último la poca capacitación y actualización relacionada a sus funciones laborales (Lozano, 2013; El economista, 2018; Rodríguez,2019).

Las rotaciones de los empleados en las organizaciones generan altos costos y concibe costos ocultos como la pérdida de la productividad y la inseguridad en los puestos de trabajo (Matthew O'Connell, y Mei-Chuan, 2007). Además, eleva los gastos relacionados con el reclutamiento y la capacitación de nuevos empleados, (Zeeshan, Sarwat, Mishal, Imam, Muhammad, 2016)

Las empresas en el Perú experimentan una crisis excesiva de rotación en su personal, situación que genera egresos innecesarios, considerándosele como el tercer país con mayor rotación de personal en América latina (PerúRetail, 2017). Todo apunta a que las empresas peruanas están realizando una deficiente selección del talento; por tal motivo, las organizaciones luchan por disminuir la rotación de empleados, ofreciendo mejoras en el ámbito laboral, y a la vez, mejoras profesionales a su personal que con el tiempo favorecerá también a la organización. Por último, las grandes organizaciones del país están capacitando a sus líderes con el fin de lograr una inspiración en su propio equipo, puedan manejar conflictos con asertividad, brindar seguridad y tolerancia ante la incertidumbre y a la vez alinearlos en el cumplimiento de la visión y misión de la empresa (Lupano y Castro, 2013).

Se detectó como problema general una alta rotación del personal en una empresa de servicios generales, lo cual ha generado una gran preocupación en la parte gerencial y 
administrativa, al no tener una solución inmediata. Este problema se refleja en los siguientes aspectos: Primero, el perfil del trabajador no está acorde a las necesidades de la empresa; segundo, la demora del proceso de incorporación y registro en la planilla de trabajo; y por último, la falta de compromiso laboral por parte de los colaboradores con la organización.

Solís (2015) en su trabajo de investigación sobre la selección y rotación de personal en base a competencias, evidenció que no existe un reclutamiento adecuado del talento humano, ya que padece de aplicaciones o etapas para este proceso como pruebas psicológicas y técnicas, que permitirán filtrar y seleccionar a los trabajadores más talentosos. Concluyó que al no contar con un proceso adecuado de reclutamiento e inducción la organización genera un alto índice de rotación del talento humano.

Por otro lado, Flores (2016) en su estudio sobre efectos en la utilidad por causa de la rotación de empleados, comprobó que la rotación se genera por el mal clima organizacional y la falta de capacitación de los empleados, que afecta seriamente la utilidad de la caja municipal. Finalmente, el autor pudo concluir que la alta rotación si afecta considerablemente a la organización en cuanto a la utilidad que genera, por la misma razón que la mayor parte de los colaboradores que se desvinculan de la organización, presentan altos conocimientos y al momento de ser remplazado, la caja de Municipal invierte nuevamente en capacitaciones para los trabajadores ingresantes.

Se puede considerar entonces que la rotación del personal, consiste en la desvinculación de un empleado con una organización, de forma voluntaria o involuntaria, pero de manera permanente. Es la proporción de los colaboradores que se retiran de una empresa en un periodo determinado, siendo este de mayor incidencia anual (Robbins y Coulter,2005; Newstrom, Davis, 2002). De igual forma, la rotación del personal, se define como el intercambio de sujetos que se retiran por diversas razones y los nuevos empleados que se incorporan a la empresa (Chiavenato,2000). Esto se representa en la siguiente figura: 


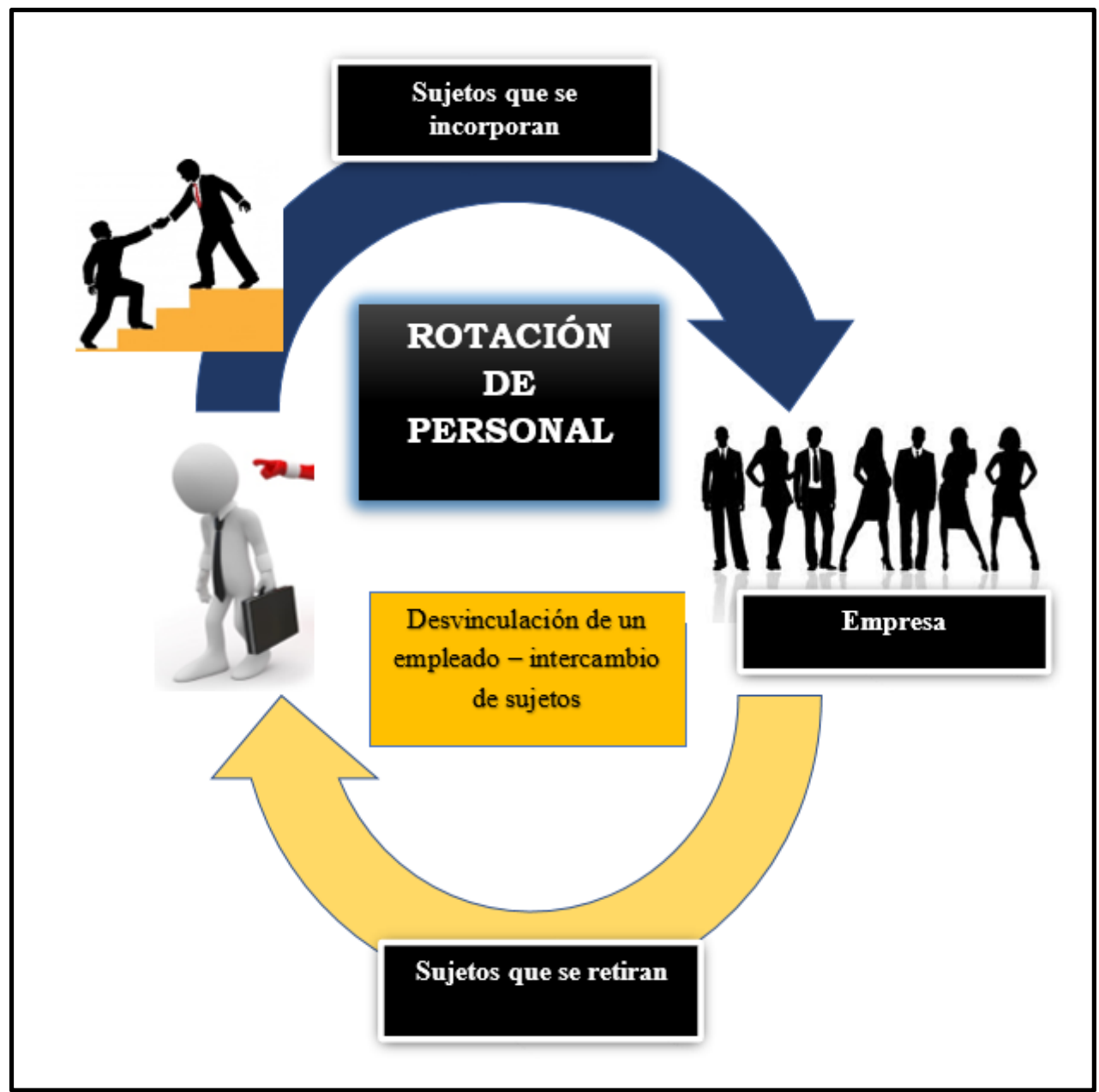

Ilustración 1. Ciclo ilustrativo de la rotación de personal. Elaboración: Nolazco (2019).

La disminución de la rotación del personal en la empresa de servicios generales es de vital importancia, y por ello es necesario estrategias de motivación y satisfacción que permitan a los trabajadores escalar en el triángulo de las necesidades de Maslow, consolidándose una estabilidad laboral que no afecte el desempeño y el desenvolvimiento del talento de los trabajadores; mitigando así el exceso de rotación. La responsabilidad para incorporar y poner en práctica estas estrategias en las políticas de la empresa, recae en los líderes directivos, porque ellos son los responsables de tomar estas decisiones para conseguir el cambio y mejora de la organización (Flores, Abreu y Badii,2008; Chiavenato, 2014).

\section{Objetivos}

Analizar la rotación del talento humano en una empresa de servicios generales de Lima, Perú.

Explicar los principales factores que determinan la rotación del talento humano en una empresa de servicios generales, Lima 2019.

Proponer un modelo basado en tres pilares para la retención del talento humano. 


\section{Método}

El estudio se realizó en una empresa de servicios generales en Lima capital del Perú, basada en un sintagma holístico y enfoque mixto (Hernández, Fernández, \& Baptista, 2010); estructurado en un nivel comprensivo, con sus procesos integrados (Hurtado, 2000). A su vez fue de tipo proyectiva, que se sostiene a través de un diagnóstico de contexto para diseñar una propuesta factible (Hurtado,2010; Carhuancho, Nolazco, Sicheri, Guerrero y Casana, 2019). Se ampara además en un método inductivo - deductivo para la información y comunicación de los resultados interpretados y analizados por el investigador (Cegarra, 2012; Romeu, 1999). Se ejecutó un análisis documentario del "seguro complementario de trabajo de riesgo" del periodo 2015-2018, brindado por la entidad en estudio, asimismo se efectuaron entrevistas a siete personas de los cuales, tres fueron ex trabajadores, tres trabajadores que laboran actualmente y al gerente general.

\section{Resultados}

\section{Resultados cuantitativos}

Una vez realizado los análisis documentarios del seguro complementario de trabajo de riesgo (SCTR) facilitados por la empresa de servicios generales, se evidencio una alta rotación del personal como muestra la siguiente tabla y figura:

Tabla 1.

Rotación del personal calculado por semestres en el periodo 2015-I al 2018-II)

\begin{tabular}{|c|c|c|c|c|c|}
\hline Año & $\begin{array}{l}\text { Promedio de personal } \\
\text { por semestre }\end{array}$ & $\begin{array}{l}\text { Total personal } \\
\text { saliente }\end{array}$ & $\begin{array}{l}\text { Total personal } \\
\text { ingresante }\end{array}$ & $\begin{array}{l}\text { Total personal } \\
\text { saliente } \%\end{array}$ & $\begin{array}{l}\text { Total Personal } \\
\text { ingresante \% }\end{array}$ \\
\hline $2015-$ & 7 & 9 & 9 & $129 \%$ & $129 \%$ \\
\hline II $2015-$ & 12 & 14 & 15 & $117 \%$ & $125 \%$ \\
\hline $2016-$ & 9 & 8 & 11 & $89 \%$ & $122 \%$ \\
\hline${ }_{\text {II }}^{2016-}$ & 11 & 12 & 12 & $109 \%$ & $109 \%$ \\
\hline $2017-$ & 13 & 9 & 11 & $69 \%$ & $85 \%$ \\
\hline${ }_{\text {II }}^{2017-}$ & 15 & 17 & 20 & $113 \%$ & $133 \%$ \\
\hline $2018-$ & 16 & 9 & 8 & $56 \%$ & $50 \%$ \\
\hline${ }_{\text {II }}^{2018-}$ & 18 & 8 & 10 & $44 \%$ & $56 \%$ \\
\hline Suma & & 86 & 96 & & \\
\hline
\end{tabular}




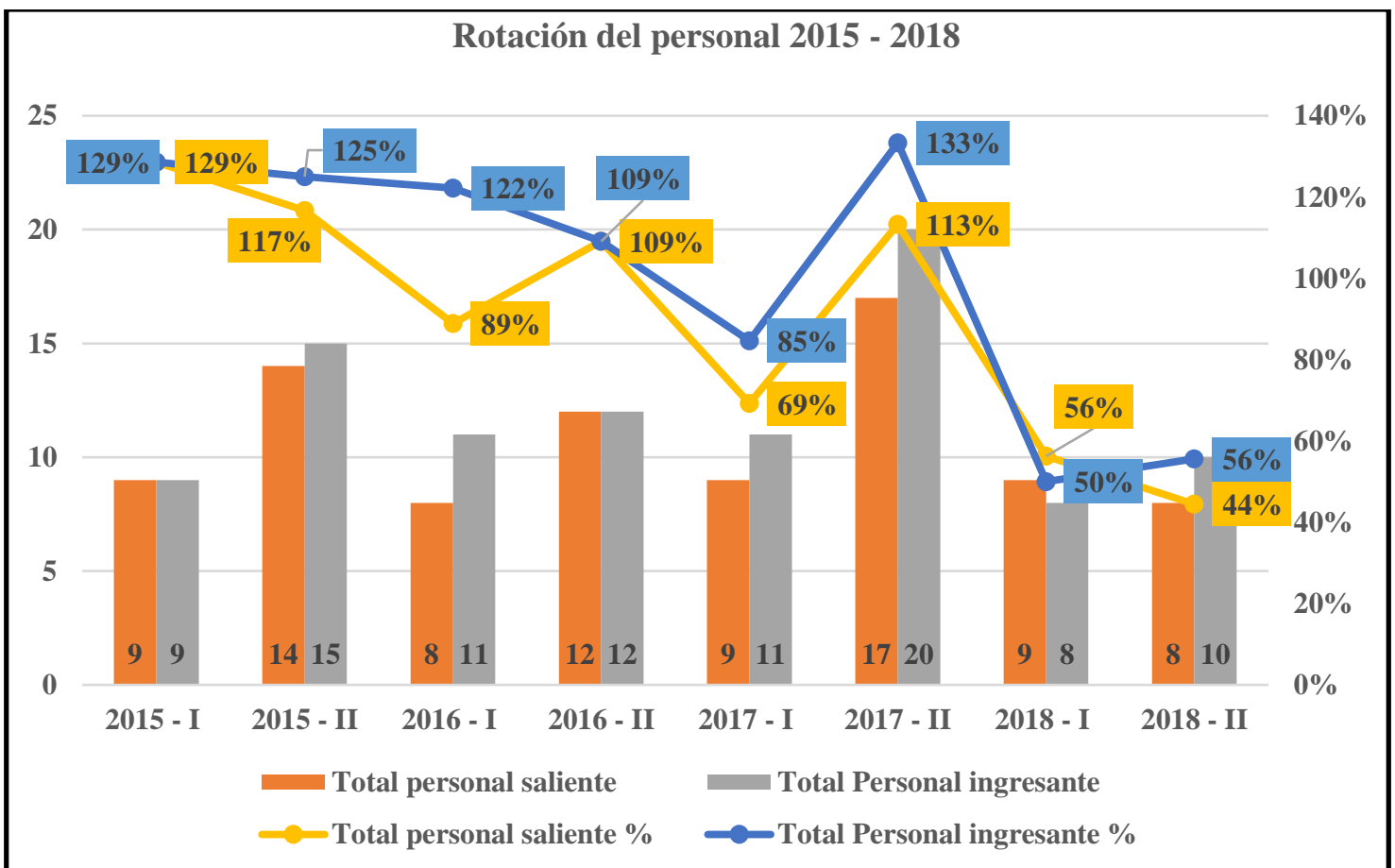

Ilustración 2. Número total de trabajadores salientes e ingresantes por cada semestre desde el 2015-I hasta el 2018II.

En la tabla 1 e ilustración 2, se visualiza la rotación del personal con sus porcentajes de manera semestral, el cual se observa que el 2017-II es el semestre de mayor rotación del personal alcanzando 17 trabajadores cesados y 20 nuevos trabajadores que cubrieron dichas vacantes, siendo equivalentes a un $113 \%$ de cesados y $133 \%$ de trabajadores nuevos. El segundo semestre de mayor rotación es el 2015-II, con 14 trabajadores cesados equivalente al $117 \%$ en base al número de trabajadores mensuales que requiere la empresa y 15 trabajadores nuevos que cubrieron vacantes, siendo este un $125 \%$ más que el promedio de trabajadores que laboran en la organización.

\section{Resultados cualitativos}

La entrevista realizada al gerente de la empresa de servicios generales fue en relación a la renuncia voluntaria e involuntaria que atraviesa su organización, respecto a la remuneración laboral: ¿Qué opinión mantiene sobre la remuneración que usted le brinda a su personal?, La remuneración en la empresa de servicios generales está acorde al mercado y considero que es una remuneración promedio ya que el horario de trabajo en la empresa es de turno noche, respecto a la oportunidad laboral: ¿Usted de qué manera les brinda oportunidades laborales? ¿Qué sugiere?, la empresa se enfoca en aquellas personas que no tiene una experiencia laboral en su trabajo o quizá no obtuvieron experiencia en el rubro al que se dedica la empresa, lo que hacemos es reclutar gente que quizá no tenga esa experiencia pero sí que tenga la capacidad para ejecutar cualquier trabajo, es decir la empresa lo que busca en este momento es reclutar a la personas que tenga esas aganas de trabajar, en cuanto a la experiencia laboral: ¿De qué manera usted reconoce la experticia laboral en sus colaboradores?, El 90\% del personal no son sujetos que tuvieron experiencia o que vinieron de otras empresas con buenos conocimientos, lo que la entidad realiza en este caso es valorar aquellas personas que tienen la capacidad para ejecutar 
sus labores o la capacidad de querer aprender. Se tiene el $10 \%$ de personal con amplia experiencia y que ha venido de otras empresas. Referente a la adaptabilidad laboral: ¿Qué opinión tiene usted sobre la adaptabilidad laboral de sus colaboradores con la empresa ¿Qué sugiere?, Conseguir que el personal se adapte al ritmo laboral de la empresa es complicado, sobre todo por el tema de descanso ya que cambiarle a una persona el ritmo de vida que tiene no es fácil por los horarios ya establecidos y la rutina diaria. Con énfasis en la seguridad laboral: ¿Qué brinda y que no brinda a su personal para logar una seguridad laboral? ¿Qué sugiere?, ¿Cómo calificaría la seguridad laboral de su empresa? Se indica que la empresa mantiene a su personal afiliado a un seguro, puesto que por lo general los trabajadores están expuestos a los accidentes, para ello la empresa les brinda el seguro complementario de trabajo de riesgo (SCTR) que cubre cualquiera eventualidad o accidente laboral. Por último, con respecto a la flexibilidad funcional: ¿Qué tanto influye la flexibilidad funcional en la rotación de su personal?, el valor que se genera en cada actividad tiene un propósito, como la empresa no vende un producto si no un servicio, la calidad del mismo se tiene que reflejar en la mano de obra efectiva, entonces todas aquellas actividades que se le encomienda al personal se tiene que realizar al pie de la letra y con mucha dedicación, para así lograr un trabajo o servicio de calidad.

En las entrevistas realizadas a los tres trabajadores que en la actualidad laboran en la empresa de servicios generales se pudo evidenciar lo siguiente: con respecto a la remuneración laboral: ¿Qué tanto influye la remuneración económica para que usted deje de laboral en la organización?, los tres trabajadores mencionaron estar conforme con el sueldo que la empresa les brindaba, pero enfatizaron que un aumento en su remuneración será crucial para que trabajan motivados ya que algunos trabajan en turno noche y es más dificultoso; y por derecho requieren un pago extra o adicional según la leyes laborales. En relación a la oportunidad laboral: ¿usted dejaría la organización por una oportunidad laboral? ¿Por qué? ¿Qué sugiere?, en este caso los entrevistados mencionaron que sí dejarían la organización si encuentran mejores beneficios, porque cada persona siempre tiene que buscar lo que le conviene recalcando una mayor remuneración y el brindarles oportunidades de crecimiento profesional; en referencia a la experiencia laboral: ¿En la empresa consideran importante tu experiencia laboral? se afirma en algunos casos que los trabajos que se ejecutan no siempre son efectivos, dado la poca experiencia de los empleados, recibiendo llamadas de atención porque se corre el riesgo que el cliente no pague, sin tener consideración en las acciones que el empleado realiza para cumplir con el servicio y evitar o enmendar cualquier falla o error . Correspondiente a la adaptabilidad laboral: ¿usted se adapta al ritmo laboral de la organización? ¿Por qué? ¿Qué sugiere?, al principio cuesta adaptarse al ritmo de la empresa que es turno noche, pero a medida del tiempo, la responsabilidad y compromiso laboral, el sujeto se acostumbra hasta que se adapta a ese ritmo, inclusive no siente mucho cansancio. Con respecto a la seguridad laboral: ¿Qué brinda y que no brinda la empresa para logar una seguridad laboral? ¿Qué sugiere? ¿Cómo calificaría asegurad laboral de la empresa?, La empresa en cuestión brinda todo lo que un trabajador necesita, está asegurado y en planilla, pero se sugiere que la empresa debería de mejorar mucho en el compromiso de todos los compañeros del trabajo, que se realice más reuniones para saber qué es lo que está pasando por que si mi compañero que trabaja en otro local y no sabe qué solución tomar, en la reunión podemos opinar todos para poder dar una solución porque para eso estamos no solo para recibir órdenes y sobre la flexibilidad funcional. Finalmente, a la pregunta: ¿Qué tanto influye la flexibilidad funcional en la rotación del personal?, La empresa quiere que 
todo se haga como ellos desean y si se cambia algunas cosas o toma de decisiones, queda bajo la responsabilidad de los empleados asumiendo los costos y perjuicios.

En relación a los tres trabajadores que ya no laboran en la empresa, manifestaron en relación a la remuneración laboral: ¿Qué opinión mantiene sobre la remuneración que percibía en la empresa?, los trabajadores manifestaron que no sentían conformes con el sueldo que la empresa me daba, porque era menos del básico, para esos entonces, además los pagos eran con recibo por honorario y si no tenías alguno no te pagaban hasta que les entregues un recibo por honorario, también me fui por que las tareas encomendadas eran muy sobrecargadas y para el sueldo no me convenía. En cuanto a la oportunidad laboral: ¿A usted de qué manera les brindaba la oportunidad laboral? ¿Qué sugiere?, en este caso los trabajadores mencionaron que no se sentían conforme con el clima laboral y la pésima infraestructura que en esos entonces se encontraba la empresa y la parte administrativa, a ello se sumaba la mala paga, todos esos factores generaron que vea oportunidades en otras organizaciones que den mejores beneficios, en referencia a la experiencia laboral: ¿De qué manera usted fue reconocido en cuanto a su experticia laboral?, Se afirma que no se valora la experiencia laboral, existen muchos novatos, que fallan en el servicio, y las personas de experiencia son pocas, enfatizando que la mayoría de expertos ya no laboran. Con respecto a la adaptabilidad laboral: ¿Qué opinión tiene usted sobre la adaptabilidad laboral en dicha empresa?, ¿Qué sugiere?, Se indica que las personas no se adaptan al ritmo de trabajo porque no está bien organizada la empresa, no se indican las formas de cómo realizar los trabajos, no se explican los procesos y las funciones específicas que cada trabajador debe realizar. En relación a la seguridad laboral: ¿Qué les brindo y que no les brindo para logar una seguridad laboral? ¿Qué sugiere? ¿Cómo calificaría asegurad laboral de dicha empresa?, No existía una buena seguridad por la misma infraestructura ya que para poder llegar a las oficinas se tiene que trasladar por los estantes de herramientas, con caminos muy angostos, a ello se suma que algunos no tenían seguro, dado que cobraban con recibo por honorarios. Finalmente, en cuanto a la flexibilidad funcional: ¿Qué tanto influyó la flexibilidad funcional que para que deje de laborar en la empresa?, los entrevistados afirman que dejaron de laboral porque $\mathrm{n}$ había flexibilidad, existía una sobrecarga laboral, con trabajos fuera de hora sin reconocimiento alguno.

\section{Modelo de los tres pilares para la retención del talento humano}

La inserción de los tres pilares en la empresa de servicios generales, se sostienen con el cumplimiento de los siguientes objetivos: Diseñar el perfil de los puestos de trabajo, diseñar un nuevo proceso de contratación y elevar el involucramiento del personal, con la finalidad de reducir la rotación del personal en la organización. 


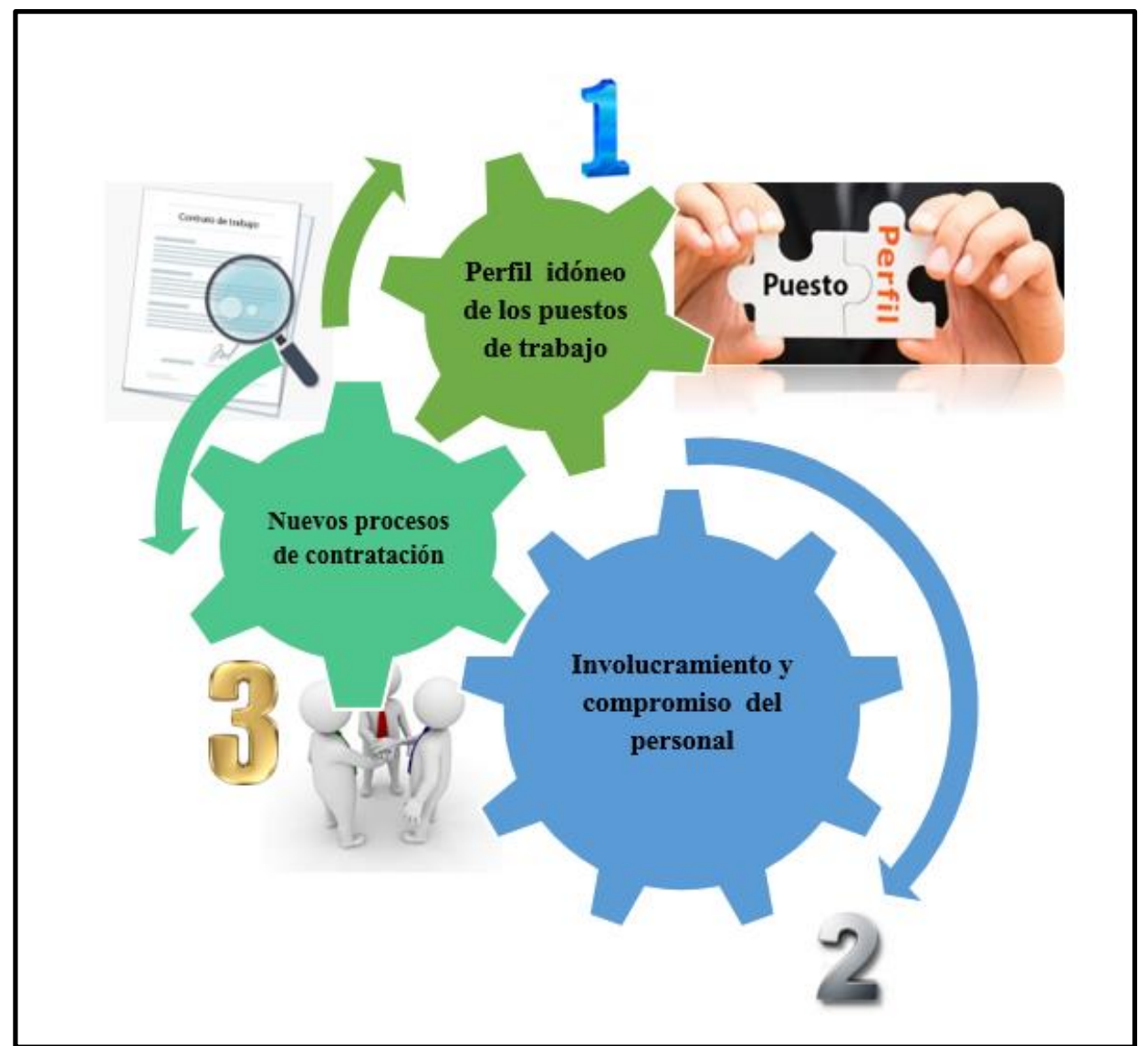

Ilustración 3. Pilares para la retención del talento humano. Elaboración: Nolazco (2019).

Pilar 1: Diseñar el perfil de los puestos de trabajo.

Actividades:

- Implementar el perfil de puestos de trabajo en el proceso de contratación.

- Realizar charlas que potencialicen la importancia del perfil ideal para los puestos de trabajo.

- Monitorear al personal ingresante en base al perfil del puesto de trabajo.

- Evaluar los resultados.

- Analizar y mejorar el perfil de los puestos de trabajo con opiniones de expertos en materia laboral.

- Considerar la opinión del personal con experiencia.

- Exponer el nuevo perfil de puestos de trabajo a la administración.

Pilar 2: Diseñar un nuevo proceso de contratación.

\section{Actividades:}

- Análisis de los procesos de contrataciones anteriores.

- Fundamentar un nuevo proceso de contratación 
- Exponer el nuevo proceso de contratación a la administración.

- Aplicación del nuevo proceso de contratación.

- Control y monitoreo del proceso de contratación.

- Evaluación de los resultados.

- Analizar y mejorar las deficiencias existentes.

- Exponer del proceso mejorado de contratación.

Pilar 3: Elevar el involucramiento y compromiso del personal.

Actividades.

- Charlas de sensibilización e inducción al personal.

- Talleres motivacionales y de identidad institucional.

- Presentación del modelo Canvas a los trabajadores.

- Evaluación a los participantes de la exposición.

- Implementación del modelo en la organización.

- Monitoreo y control del modelo Canvas.

- Evaluar los resultados.

- Implementar mejoras del modelo.

- Aplicación del modelo renovado en la organización.

El modelo Canvas es un instrumento para diseñar y concretar modelos de negocio e innovación trabajados estratégicamente en cuatro áreas: clientes, oferta, infraestructura y viabilidad económica en base a sus estrategias que toma en consideración: Segmento de clientes, propuesta de valor, canales, relación con los clientes, flujos de ingresos, recursos, actividades, aliados y estructura. Esta metodología, es una de las mejores herramientas de la organización y se utiliza para explicar fácilmente a un grupo de trabajadores, en qué consiste la idea de negocio y que es lo que busca la organización en función al desempeño, funcionalidad y retención de los colaboradores.

\section{Conclusiones}

Con la finalidad reducir la rotación del talento humano que presenta la organización se presentó los tres pilares para la retención del talento humano en la organización los cuales son: Diseñar el perfil de los puestos de trabajo, diseñar un nuevo proceso de contratación y elevar el involucramiento y compromiso del personal.

Para efectuar el diagnóstico final se tuvo que recopilar datos cuantitativos y cualitativos para luego poder procesarlas con la ayuda del Software Atlas ti, con la finalidad de detectar a profundidad los problemas presentados expuestos en este estudio; concluyéndose que la alta rotación que presenta la organización es debido a que el perfil del trabajador no está acorde a las necesidades de la empresa, que la demora del proceso de incorporación a la planilla de trabajo genera disconformidad e inseguridad de permanencia y una falta de compromiso del personal con la organización. 
La propuesta de solución consiste en implantar los tres pilares para la retener del talento humano en la empresa de servicios generales, enfocadas a reducir la rotación del personal, aumentar la satisfacción laboral y generar una integración eficiente del trabajador con la empresa.

\section{Bibliografía}

By Matthew O'Connell, Ph.D. \& Mei-Chuan Kung. (2007). Employee Turnover \& Retention: Understanding the True Costs and Reducing them through Improved Selection Processes. ResearchGate, 1, 10.

Carhuancho, I., Nolazco, F., Sicheri, G., Guerrero, M. y Casana, K. (2019). Metodología de lainvestigación holística. Ecuador: UIDE.

Cegarra, J. (2012). Los métodos de investigación científica y tecnológica. Madrid: Díaz de Santos.

Chiavenato, I. (2000). Administración de Recursos Humanos. Colombia: McGraw-Hill.

Chiavenato, I. (2012). Introducción a la teoría general de la administración. México: Mc GrawHill.

Chiavenato, I. (2014). Introducción a la teoría general de la administración. México: McGrawHill.

Economista, E. (11 de julio de 2018). Rotación de personal: los empleados no solo se marchan por dinero. El economista. https://factorcapitalhumano.com/mundo-del-trabajo/rotacionde-personal-los-empleados-no-solo-se-marchan-por-dinero/2018/07/

Flores, J. (2016). Rotación de empleados y su efecto en la utilidad de la caja municipal de ahorro y crédito Arequipa en el periodo 2011 - 2015. Moquegua: Universidad José Carlos Mariátegui.

Flores, R., JAbreu, J. y Badii, M. (2008). Factores que originan la rotación de personal en las empresas mexicanas Revista Daena: International Journal of Good Conscience. 3(1): 65-99. Marzo 2008. ISSN 1870-557X.

Hernández, R., Fernández, C., \& Baptista, M. (2010). Metodología de la investigación. México: McGraw-Hill Educación.

Hurtado, J. (2000). Metodología de la investigación holística. Caracas: Sypal.

Hurtado, J. (2010). Guía para la comprensión holística de la ciencia. Caracas: Sypal.

Lozano, L. (2013). El talento humano una estrategia de éxito en las empresas culturales. Revista Escuela de Administración de Negocios, (60), 147164. https://doi.org/10.21158/01208160.n60.2007.409

Lupano, M., y Castro, A. (2013). Estudios sobre el liderazgo. Teorías y evaluación. Psicodebate 6. Cultura y Sociedad, 107-121. http://www.palermo.edu/cienciassociales/publicaciones/pdf/Psico6/6Psico\%2008.pdf

Newstrom, J. y Davis, K. (2002). Comportamiento humano en el trabajo. México: McGraw-Hill / Interamericana.

PerúRetail. (10 de 03 de 2017). La rotación de personal no deseada es la que sin duda acarrea costos. La rotación de personal no deseada es la que sin duda acarrea costos, (Zeeshan , Sarwat, Mishal, Imam , \& Muhammad, 2016)

https://www.peru-retail.com/rotacion-de-personal-no-deseada-acarrea-costos/?

Robbins, S., y Coulter, M. (2005). Administración. México: Pearson Educación.

Rodríguez, D. (2019) Estrategias para retener el talento humano en una empresa de servicios generales, Lima 2019. (tesis de licenciatura). Universidad Norbert Wiener, Lima, Perú. 
Romeu, E. (1999). Aplicación del enfoque comunicativo en la escuela media. La Habana: Pueblo y Educación.

Solís, D. (2015). Selección de personal por competencia y rotación de personal en los colaboradores de la empresa Repremarva de la ciudad de Ambato provincia de Tungurahua. (tesis de licenciatura) Ecuador: Universidad técnica de Ambato.

Zeeshan , A., Sarwat, S., Mishal, K., Imam , A., \& Muhammad, A. (Noviembre de 2016). Impact of Employee Turnover on Organisational Effectiveness in Tele Communication Sector of Pakistan. IOSR Journal of Business and Management (IOSR-JBM), 18, 88-96. doi:10.9790/487X-1811058896 